\title{
A case report of asymptomatic long-segmental aortic dissection diagnosed by transthoracic echocardiography
}

\section{Jiwei Wang}

The Second Affiliated Hospital of Nanchang University https://orcid.org/0000-0002-6202-4652

Bin Lai

The Second Affiliated Hospital of Nanchang University

Cai Yao

The Second Affiliated Hospital of Nanchang University

\section{Yongbing Wu}

The Second Affiliated Hospital of Nanchang University

\section{Yanna Liu ( 0 527161577@qq.com )}

The Second Affiliated Hospital of Nanchang University

\section{Case report}

Keywords: Painless, Asymptomatic, Aortic dissection, Transthoracic echocardiography

Posted Date: November 15th, 2019

DOI: https://doi.org/10.21203/rs.2.17293/v1

License: (9) This work is licensed under a Creative Commons Attribution 4.0 International License. Read Full License 


\section{Abstract}

Background: Aortic dissection (AD) is a life-threatening disease with high mortality rate. Severe pain in chest, back or abdomen is the most common symptom. Painless, but with a variety of other symptoms, also happened in some AD patients. Asymptomatic AD is exceptionally rare and often under-recognized.

Case presentation: A 51-year-old man presented to cardiovascular department accompanied with an exaggerated low DBP and widened PP when measuring routine BP. Blood pressure was $124 / 36 \mathrm{mmHg}$ (PP $88 \mathrm{mmHg}$ ) in his right arm and $108 / 32 \mathrm{mmHg}$ (PP 76mmHg) in his left arm. Transthoracic echocardiography was scheduled and showed that dissection intimal flaps are visualized in the aortic root, aortic arch and descending aorta. Subsequent CT angiography (CTA) was performed and demonstrated that a long-segmental AD occurred from aortic root to left common iliac artery. The patient underwent replacement of the aortic root, ascending aorta, and aortic arch with endovascular stentgraft placement into the descending aorta. At three months of follow-up, he was asymptomatic and with no signs of target organ damage.

Conclusions: A careful TTE scan is particularly important for asymptomatic AD patient because it most likely as a routine imaging technique used for cardiovascular evaluation. If miss-diagnosed and underrecognized by clinician, untreated patients with prolonged dissection will become highly susceptible to an aortic rupture or ischemia to organs and leads to mortality.

\section{Background}

Aortic dissection (AD) is a catastrophic medical emergency, which leads to severe morbidity and high probability of mortality. Typically, AD patients present with severe pain in chest, back, or abdomen ${ }^{[1]}$. Painless $A D$ has been report in some cases, but usually with a wide variety of other symptoms, including syncope ${ }^{[2]}$, paraplegia ${ }^{[3]}$, amnesia ${ }^{[4]}$, dyspnea ${ }^{[5]}$, nausea, vomiting ${ }^{[6]}$, Dysphagia, hoarseness ${ }^{[7]}$, vertigo

${ }^{[8]}$, and so on. Asymptomatic AD is extremely rare. It has been reported occasionally and was diagnosed mostly by transesophageal echocardiography (TEE) or contrast computed tomography (CT) ${ }^{[9-12]}$. We will discuss a case of long-segmental $A D$ without any obvious symptom, with which, the patient presented to cardiovascular department complaining of exaggerated low diastolic blood pressure (DBP) and widened pulse pressure (PP) when measuring routine blood pressure (BP).

\section{Case Report}

A 51-year-old man presented to cardiovascular department accompanied with an exaggerated low DBP and widened PP when measuring routine BP. He had hypertension for more than twenty years and received regular medications. He reported smoking one pack per day during the last 25 years.

Physical examination showed a diastolic murmur of grade $3 / 6$ on the left sternal edge. Blood pressure was $124 / 36 \mathrm{mmHg}$ (PP $88 \mathrm{mmHg}$ ) in his right arm and 108/32 mmHg (PP 76mmHg) in his left arm. 
Heart rate was 79 beats per minute. He had no Marfanoid appearance. He denied any symptoms. Therefore, he was suggested to take an electrocardiogram(ECG) and transthoracic echocardiography (TTE) examination.

The ECG revealed low voltage of the QRS complexes in the limb leads with nonspecific T-wave abnormality. The TTE in left parasternal view showing dissection intimal flap originated in aortic root (Figure 1A, Video1A). On Color Doppler examination, there was moderate aortic regurgitation. The left ventricular was mild dilated with an ejection fraction of $69 \%$ and diastolic dysfunction. In the suprasternal view showing Intimal flap propagated to was the aortic arch (Fig 1B, Video1B). Abdominal vascular ultrasound showing intimal flap extended to abdominal aorta artery (Fig 1C, Video1C), Color Doppler showing there was a flow from the true lumen $(T L)$ to false lumen $(F L)$ in the systole (Fig 1D, Video1D). Subsequent CT angiography (CTA) was performed and demonstrated that a Stanford type A

aortic dissection that originates in ascending aorta, propagates to aortic arch, descending thoracic aorta, descending abdominal aorta and left common iliac artery (Figure $2 \mathrm{~A}-\mathrm{F}$ ).

The patient underwent replacement of the aortic root, ascending aorta, and aortic arch with endovascular stent-graft placement into the descending aorta. At three months of follow-up, he was asymptomatic and with no signs of target organ damage.

\section{Discussion}

$A D$ is a life-threatening emergency accompanied with high mortality rate. It is also associated with hypertension and genetic connective tissue abnormalities that may culminate in medial degeneration, intimal tear and false lumen formation. There are two well-known classifications of AD (Figure 3), the Stanford type A and $B^{[13]}$, and the DeBakey type I - III ${ }^{[14]}$. The Stanford type A refers to the dissections which involve the ascending aorta and may extend to the aortic arch and descending aorta. In the contrast, the type B only involve the descending aorta. DeBakey type I refers to the dissections which involves the whole aorta, however, the type II only involve the ascending aorta, and the type III only involves the descending aorta. Type III further subdivided into IIIa and IIIb. IIla limits to descending thoracic aorta and IIIb extends below diaphragm. Therefore, Stanford type A dissection includes DeBakey types I and II, and Stanford type B includes DeBakey type IIla and type IIIb. According to the classification mentioned above, our case belongs to Stanford type A dissection or DeBakey type I.

Acute sudden onset of severe pain in the chest, back or abdomen is the typical manifestation of AD. However, $5 \%$ to $15 \%$ of $A D$ patients present painless ${ }^{[15,16]}$, but with a wide variety of other symptoms, including syncope, paraplegia, amnesia, dyspnea, nausea, vomiting, Dysphagia, hoarseness, vertigo, and so on. Asymptomatic AD is extremely rare. It has been reported occasionally and was diagnosed by transesophageal echocardiography (TEE) or contrast CT. In our case, the patient is a long-segmental AD patient, but completely asymptomatic, which only showed an exaggerated low DBP and widened PP when measuring routine BP. PP more than $60 \mathrm{mmHg}$ is widened PP. It is an indicator of the structure or function change in heart; and it may be the result of severe anemia, hyperthyroidism, aortic regurgitation 
and atherosclerotic disease. Widened PP only has been reported as one of signs in AD patients $[3,17]$, however, we first found that it could be the only complain of AD patients.

The diagnosis of $A D$ is made by a variety of imaging techniques, such as TTE, TEE, CT, CTA and Magnetic resonance imaging(MRI). Due to the shorter acquisition time, wide availability, and high diagnostic accuracy, CT and CTA have been considered as the ideal methods for diagnosis and monitoring of patients with aortic disease. Furthermore, MRI has high accuracy, sensitivity and specificity equal to or superior to CT. TTE, as a routine imaging technique for cardiovascular evaluation, is usually used in AD patient as an initial method. However, the usage of TTE is limited in AD diagnosis because of an inadequate echo window and suboptimal echo quality, especially in obese patients and in those with chronic obstructive pulmonary disease. However, TTE may be the only method which will be used for asymptomatic $A D$ patients, since patients without any symptoms to attract enough attention by clinician. To reduce the risk of miss diagnosis in asymptomatic or painless $A D$, it is important to pay more attention in aortic root, ascending aorta, aortic arch and descending aorta when take the left parasternal view and suprasternal view of TTE.

\section{Conclusion}

AD patients do not always present acute chest, back, and tearing abdominal pain. Painless, but with a variety of other symptoms, also happened in some $A D$ patients. Asymptomatic $A D$ is exceptionally rare and often under-recognized. A careful TTE scan is particularly important for asymptomatic AD patient because it most likely as a routine imaging technique used for cardiovascular evaluation. If missdiagnosed and under-recognized by clinician, untreated patients with prolonged dissection will become highly susceptible to an aortic rupture or ischemia to organs and leads to mortality. To improve the diagnosis of painless or asymptomatic $A D$, more attention should be paid in aortic root, ascending aorta, aortic arch and descending aorta when take the left parasternal view and suprasternal view of TTE.

\section{Declarations}

\section{Ethics approval and consent to participate}

Not applicable

\section{Consent for publication}

All authors have read and approved the paper, and agree the author name order.

\section{Availability of data and materials}


All data generated or analyzed during this study are included in this published article. Additional data can be requested from the department of Ultrasound, The Second Affiliated Hospital of Nanchang University, Nanchang, China.

\section{Competing interests}

The authors declare that they have no competing interests.

\section{Funding}

No funding was received

\section{Authors' contributions}

JW wrote the manuscript. BL edited all pictures and drew the picture 3. CY diagnosed AD via TTE scan. YB performed the surgery for the patient. YN edited and revised the manuscript. All authors read and approved the final manuscript.

\section{Acknowledgements}

Not applicable.

\section{Authors' information}

- Jiwei Wang, MD, PhD, Department of Ultrasound, The Second Affiliated Hospital of Nanchang University, Nanchang, Jiangxi, 330006, China. Email: wangjiwei167@163.com;

- Bin Lai, MD, PhD, Department of Gastrointestinal Surgery, The Second Affiliated Hospital of Nanchang University, Nanchang, Jiangxi, 330006, China. Email: vincent83126@163.com;

- Cai Yao, MD, Department of Ultrasound, The Second Affiliated Hospital of Nanchang University, Nanchang, Jiangxi, 330006, China. Email: 57026609@qq.com;

- Yongbing Wu, MD, Department of Cardiac Surgery, The Second Affiliated Hospital of Nanchang University; Nanchang, Jiangxi, 330006, China. Email: 735063414@qq.com;

- Yanna Liu, MD, Department of Ultrasound, The Second Affiliated Hospital of Nanchang University, Nanchang, Jiangxi, 330006, China. Email: 527161577@qq.com;

\section{References}

1. Vilacosta I, San Román JA. Acute aortic syndrome. Heart. 2001 Apr;85(4):365-368.】 
2. Chen $\mathrm{CH}$, Liu KT. A case report of painless type A aortic dissection with intermittent convulsive syncope as initial presentation. Medicine (Baltimore). 2017 Apr;96(17): e6762.

3. Takeda S, Tanaka Y, Sawada Y, Tabuchi A, Hirata H, Mizumoto T. Repetitive transient paraplegia caused by painless acute aortic dissection. Acute Med Surg. 2019 Feb;6(2):188-191.

4. April MD, Fossum K, Hounshell C, Stolper K, Spear L, Semelrath K. A sinister cause of anterograde amnesia: painless aortic dissection. Am J Emerg Med. 2015 Jul;33(7):989. e5-e7.

5. Marroush TS, Boshara AR, Parvataneni KC, Takla R, Mesiha NA. Painless aortic dissection. Am J Med Sci. 2017 Nov;354(5):513-520.

6. Fatima S, Sharma K. Painless aortic dissection-diagnostic dilemma with fatal outcomes: what do we learn? J Investig Med High Impact Case Rep. 2017 Jul 31;5(3):2324709617721252.

7. Lee SI, Pyun SB, Jang DH. Dysphagia and hoarseness associated with painless aortic dissection: a rare case of cardiovocal syndrome. Dysphagia. 2006 Apr;21(2):129-132.

8. Demiryoguran NS, Karcioglu O, Topacoglu H, Aksakalli S. Painless aortic dissection with bilateral carotid involvement presenting with vertigo as the chief complaint. Emerg Med J. 2006 Feb;23(2): e15.

9. Khan IA, Vasavada BC, Sacchi TJ. Asymptomatic dissection of the ascending aorta: diagnosis by transesophageal echocardiography. Am J Emerg Med. 1999 Mar;17(2):172-173.

10. Cohen R, Mena D, Carbajal-Mendoza R, Arole O, Mejia JO. A case report on asymptomatic ascending aortic dissection. Int J Angiol. 2008 Fall;17(3):155-161.

11. Kumar A, Kumar K, Zeltser R, Makaryus AN. Nearly asymptomatic eight-month thoracic aortic dissection. Clin Med Insights Cardiol. 2016 May 25; 10:75-8.

12. Abo-Salem E, López-Candales A. Diagnosis of asymptomatic aortic dissection during pregnancy using contrast echocardiography. J Cardiol Cases. 2014 Feb 24;9(5):200-202.

13. Daily P, Trueblood H, Stinson, Wuerflein R, Shumway N. Management of acute aortic dissections. Ann Thorac Surg 1970; 10: 237-47. 『

14. DeBakey ME, Cooley DA, Creech $\mathrm{O}$. Surgical considerations of dissecting aneurysm of the aorta. Ann Surg. 1955; 142: 586-612.

15. Wang W, Duan W, Xue Y, Wang L, Liu J, Yu S, Yi D. Clinical features of acute aortic dissection from the registry of aortic dissection in China. J Thorac Cardiovasc Surg. 2014 Dec;148(6):2995-3000.

16. HIRST AE, JOHNS VJ, KIME SW. Dissecting aneurysm of the aorta: a review of 505 cases. Medicine (Baltimore). 1958 Sep;37(3):217-79.

17. Qin C, Tian DS. Acute ischemic stroke due to painless long-segmental aortic dissection. Neurology. 2019 Mar 5;92(10):484-485.

\section{Figures}




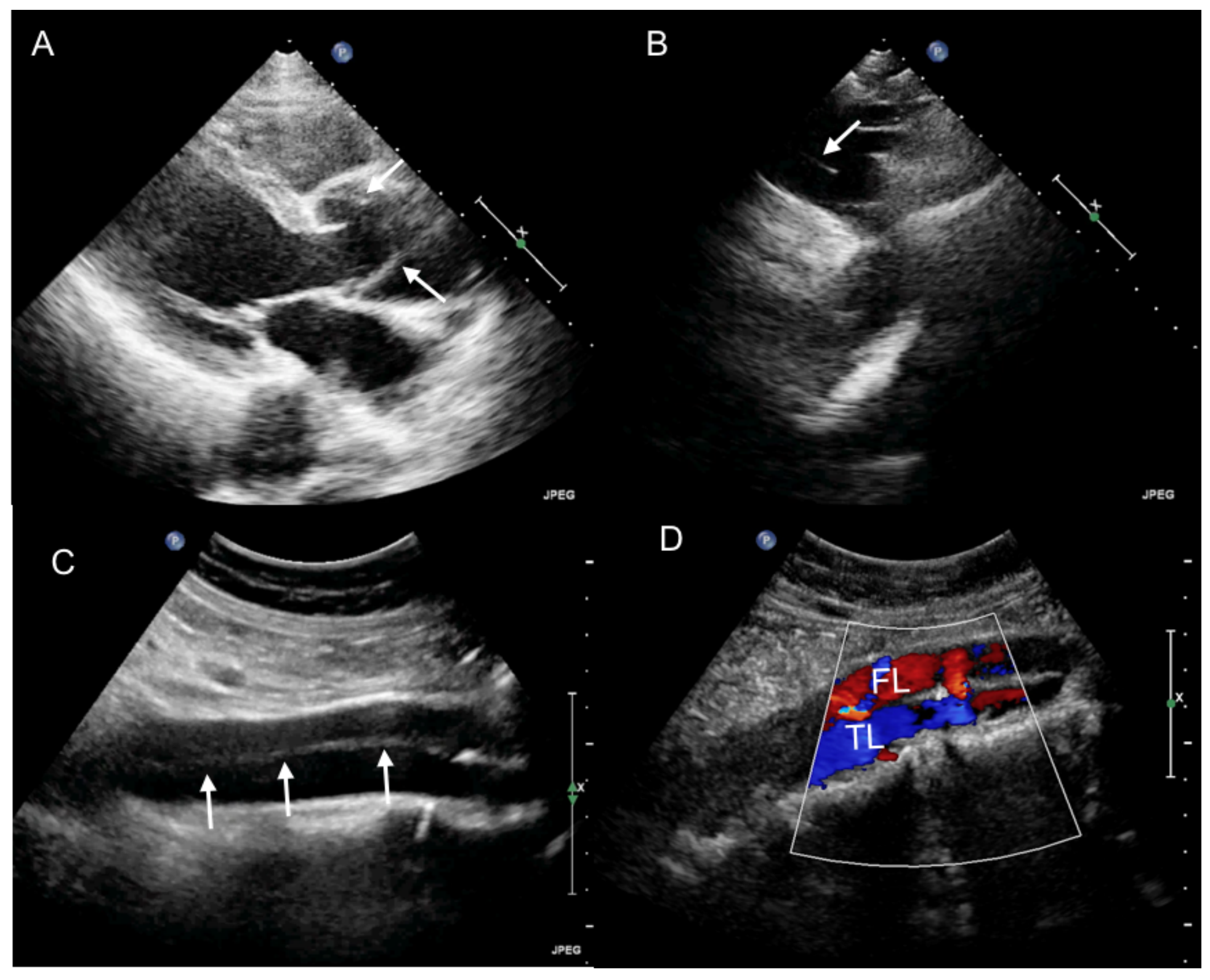

\section{Figure 1}

Type 1 aortic dissection diagnosis by transthoracic echocardiography. (A) The left parasternal view showing dissection intimal flap (arrows) originated in aortic root. (B) The left parasternal view showing intimal flap propagated to aortic arch. (C) Abdominal vascular ultrasound showing the intimal flap extended to abdominal aorta artery. (D) Color Doppler showing there was a flow from the true lumen (TL) to false lumen $(F L)$ in the systole. 


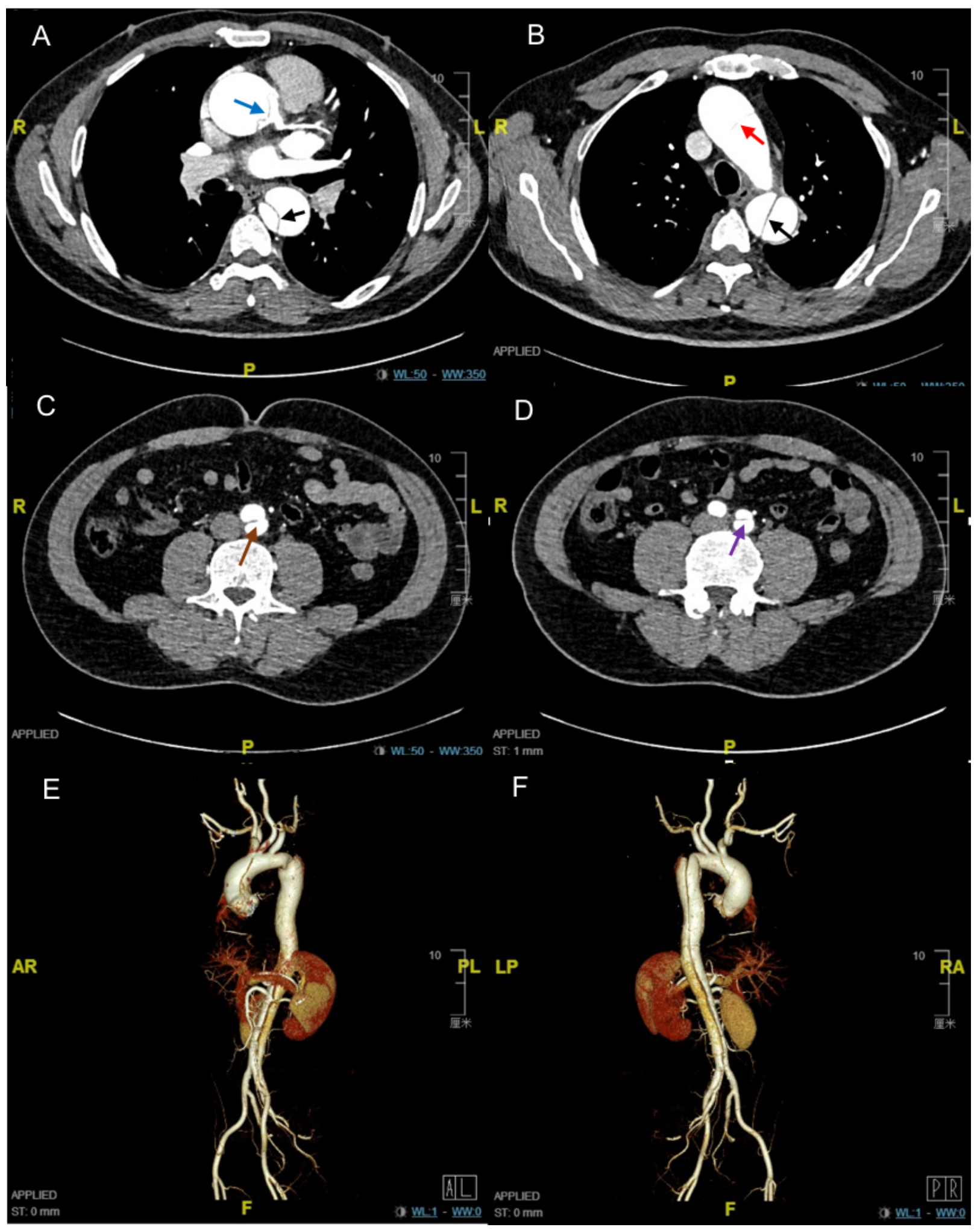

\section{Figure 2}

CTA showing Type 1 aortic dissection. (A-D) Intimal flap and two lumina are visualized in the aortic root (blue arrow), aortic arch (red arrow), descending thoracic aorta (black arrow), descending abdominal aorta (brown arrow), and left common iliac artery (purple arrow). (E-F) Three-Dimensional reconstruction showing intimal flap extended from aortic root to left common iliac artery. 


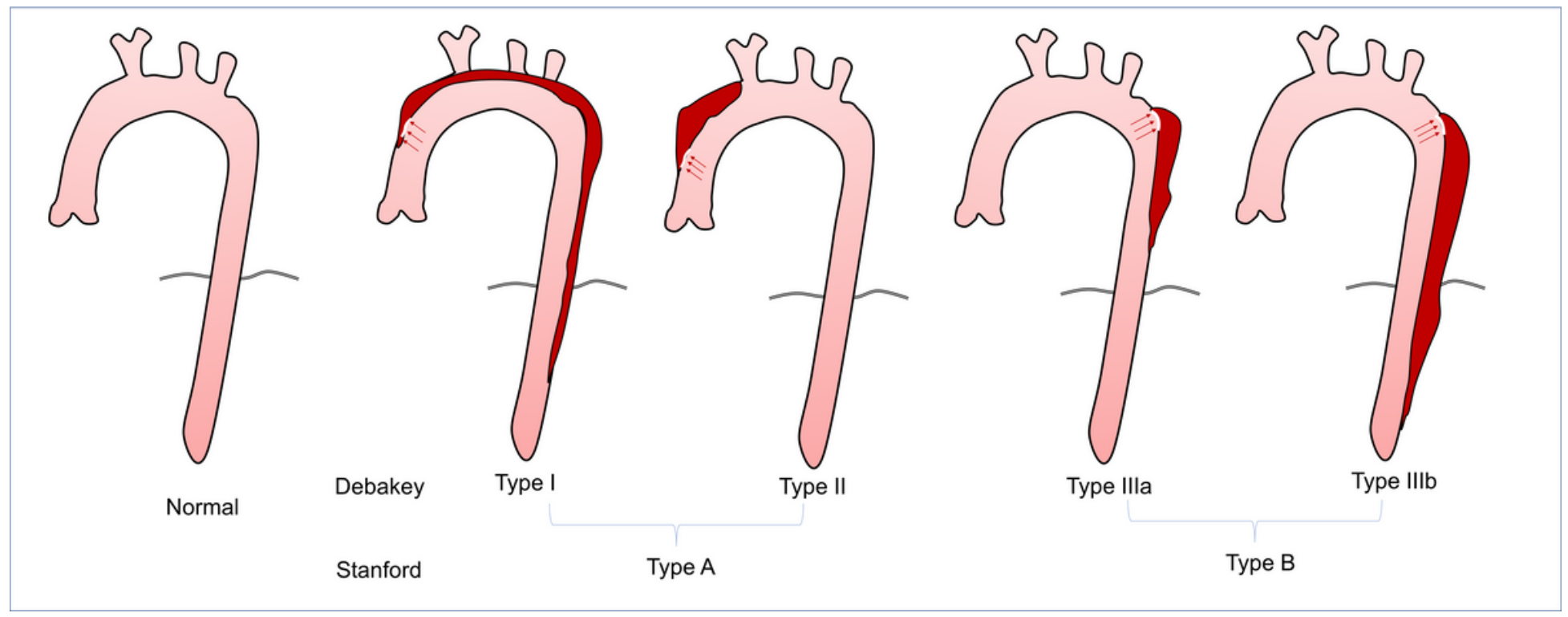

Figure 3

Classification of aortic dissection according to Stanford and DeBakey (drawn by Bin Lai).

\section{Supplementary Files}

This is a list of supplementary files associated with this preprint. Click to download.

- Video1A.avi

- Video1B.avi

- Video1D.avi

- Video1C.avi 